\title{
Doppler-estimated Carotid and Brachial Artery Flow as Surrogate for Cardiac Output: Needs Further Validation
}

\author{
Velmurugan Selvam ${ }^{1} \odot$, Shrikanth Srinivasan ${ }^{2} \odot$ \\ Keywords: Brachial artery velocity time integral, Carotid artery velocity time integral, Fluid responsiveness, Left ventricular outflow tract velocity \\ time integral. \\ Indian Journal of Critical Care Medicine (2022): 10.5005/jp-journals-10071-24108
}

The main goal of fluid administration in critically ill patients is to increase the venous return and ultimately to improve cardiac output and oxygen delivery to the tissues There is also increasing knowledge recently regarding the risks of fluid overload and its impact on organ failure, especially lung. Volume overload has been associated with significant increase in mortality in sepsis ${ }^{1}$ and increases duration of mechanical ventilation in patients with acute respiratory distress syndrome (ARDS). ${ }^{2}$ Individualized strategy of fluid management in sepsis have shown to improve outcomes in several clinical trials. ${ }^{3,4}$ Fluid responsiveness (FR) should be assessed before volume expansion to avoid volume overload and its complications.

Recent evidence shows that various dynamic parameters such as pulse pressure variation (PPV), systolic pressure variation (SPV), and stroke volume variation (SVV) induced by cyclic positive pressure ventilation have a high and specificity for predicting FR compared to static markers. ${ }^{5,6}$ In recent years, modern intensive care has focused on less-invasive and noninvasive techniques with the avoidance of unnecessary invasive procedures such as arterial line cannulation which could contribute to patient morbidity.

Transthoracic echocardiography is a reliable and noninvasive method of assessment of FR. Measurement of left ventricular outflow tract velocity time integral (LVOT-VTI), derived stroke volume (SV), and cardiac output reliably predicts FR in critically ill patients. ${ }^{7,8}$ However, there are some limitations of using echocardiography for predicting FR. The measurement of echocardiographic parameters depends on the patient's echogenicity and various factors such as obesity, chest deformity, and patient positioning may affect proper echo window in critically ill patients.

Recently, ultrasound Doppler assessment of carotid artery velocity time integral (CAVTI), carotid artery (CA) blood flow, brachial artery velocity time integral (BAVTI), and brachial artery flow has been evaluated as an alternative to LVOT-VTI and cardiac output to predict FR, as measurement of carotid artery flow and brachial artery flow is easier, does not depend on adequate cardiac windows, and requires less skill and expertise. However, these parameters have not been evaluated in larger studies.

Carotid flow, CAVTI, and brachial flow as a surrogate of cardiac output to assess FR have been evaluated in some clinical trials, which showed mixed results. Pilot study by Weber et al. ${ }^{9,10}$ found poor correlation between both carotid blood flow and brachial blood flow and cardiac index in healthy volunteers and in patients undergoing cardiovascular surgery. Authors of the study concluded
${ }^{1}$ Department of Critical Care, Sri Ramachandra Medical College and Research Institute, Chennai, Tamil Nadu, India

${ }^{2}$ Department of Critical Care Medicine, Manipal Hospital, New Delhi, India

Corresponding Author: Velmurugan Selvam, Department of Critical Care, Sri Ramachandra Medical College and Research Institute, Chennai, Tamil Nadu, India, Phone: +91 9968859560, e-mail: dr.velsri86@gmail.com

How to cite this article: Selvam V, Srinivasan S. Doppler-estimated Carotid and Brachial Artery Flow as Surrogate for Cardiac Output: Needs Further Validation. Indian J Crit Care Med 2022;26(2):159-160.

Source of support: Nil

Conflict of interest: None

that Doppler-estimated carotid and peripheral arterial blood flows cannot be used to provide noninvasive estimates of cardiac index. Similarly, in a study by Chowhan et al., ${ }^{11}$ which included 60 critically ill mechanically ventilated patients, found that following passive leg raise test (PLR), the correlation between $\triangle C A V T I$ and $\triangle$ LVOTVTI is weak in septic shock patients and only modest in sepsis patients and concluded that CAVTI cannot be reliably used as an estimate of CO to assess FR. Another human volunteer study by Sidor et al. ${ }^{12}$ found a positive correlation between carotid systolic flow and cardiac output, but the correlation between total carotid flow estimated by VTI and cardiac output was poor.

In the current study by Joshi et al., ${ }^{13}$ authors studied the correlation and agreement between VTi and flow at carotid and brachial arteries $\left(V_{T i}\right.$ TAP Carotid $_{\text {, VTi_TAP }}$ brachial, and VTi_flow Brachial $_{\text {. }}$

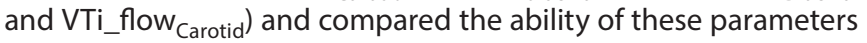
to predict FR in adults ( $>18$ years) undergoing elective, supramajor abdominal oncosurgeries under general anesthesia. Fifty instances requiring fluid boluses from 27 patients were studied. A fluid bolus of $7 \mathrm{~mL} / \mathrm{kg}$ Ringers Lactate was given over 30 min when clinically indicated. PPV was measured using Philips IntelliVue MP70 monitor. $\mathrm{SVV}, \mathrm{SV}$, and $\mathrm{Cl}$ were recorded using the $\mathrm{EV} 1000^{\mathrm{TM}}$ clinical platform. The Doppler measurements of carotid artery and brachial artery were carried out by a single investigator trained in critical care ultrasound. Patients were divided into two groups, responders and nonresponders, based on increase in stroke volume (SV) $\geq 15 \%$. Authors found weak correlation between VTi_TAP Carotid $_{\text {and }}$ VTi_TAP ${ }_{\text {Brachial }}\left(r^{2}=0.143\right)$ and VTi_flow ${ }_{\text {carotid }}$ and VTi_flow brachial $\left(r^{2}=0.0004\right)$. Also, the ability of VTi_TAP ${ }_{\text {Carotid }}, \mathrm{VTi}_{-} \mathrm{TAP}_{\text {brachial }}$,

(0) The Author(s). 2022 Open Access This article is distributed under the terms of the Creative Commons Attribution 4.0 International License (https://creativecommons. org/licenses/by-nc/4.0/), which permits unrestricted use, distribution, and non-commercial reproduction in any medium, provided you give appropriate credit to the original author(s) and the source, provide a link to the Creative Commons license, and indicate if changes were made. The Creative Commons Public Domain Dedication waiver (http://creativecommons.org/publicdomain/zero/1.0/) applies to the data made available in this article, unless otherwise stated. 
VTi_flow Brachial, $_{\text {, and VTi_flow }}$ Carotid to assess $F R$ was poor with AUROC of around 0.5-0.55. Surprisingly, the ability of PPV and SVV to predict FR was also moderate with AUROC of 0.69 and 0.63 , respectively, much less than previously documented in other studies.

The results of the study, however, should be interpreted with caution for the following reasons:

- Responders and nonresponders were not determined using a standard technique. It is important to perform fluid challenge test properly to differentiate between responders and nonresponders and to maximize the predictability of FR test. In this study, authors had given $7 \mathrm{~mL} / \mathrm{kg}$ of fluid over $30 \mathrm{~min}$ as fluid challenge, which is not ideal way of fluid challenge. Seven milliliter per kilogram of fluid (around $500 \mathrm{~mL}$ ) can itself cause fluid overload in nonresponders and also this large volume of fluid may result in positive response even in nonresponders (false positive).

- Duration of fluid challenge is important thing that can affect the results. Factors such as depth of anesthesia, patient positioning, pain, and blood loss during that 30 min duration could have influenced the results. Ideally, fluid challenge over 5-10 min duration during which other factors kept unchanged is recommended. Several others test such as passive leg raising test, mini fluid challenge (100 $\mathrm{mL}$ over $1 \mathrm{~min})$, and end expiratory occlusion test have been shown to predict FR accurately and rapidly in several recent studies over a fluid challenge over 30 min duration.

- Authors tried to find the correlation between VTi and flow at carotid and brachial arteries; however, both of them are not reliable substitute for the aortic VTI and not validated for assessing FR. Studies in healthy volunteers and cardiac surgical patients have shown a poor correlation between cardiac index and blood flow velocity in the carotid and peripheral sites.

- The sample size is too small that can compromise the conclusions drawn from the study. Small sample size may prevent the findings from being extrapolated.

Current study found weak correlation between both VTI and flow measured at carotid and brachial arteries. Till date, the existing literature does not support carotid/brachial VTI and flow as a surrogate of aortic VTI/cardiac output to assess FR in critically ill patients and patients undergoing elective surgery. Further, larger studies with standard fluid responsive test are required to test predictability of carotid and brachial VTi and flow to assess FR.

\section{ORCID}

Velmurugan Selvam @ ic https://orcid.org/0000-0002-9495-2293
Shrikanth Srinivasan $\odot$ https://orcid.org/0000-0002-5336-3767

\section{References}

1. Acheampong A, Vincent J-L. A positive fluid balance is an independent prognostic factor in patients with sepsis. Crit Care 2015;19(1):251. DOI: 10.1186/s13054-015-0970-1.

2. Rosenberg AL, Dechert RE, Park PK, Bartlett RH, NIH NHLBI ARDS Network. Review of a large clinical series: association of cumulative fluid balance on outcome in acute lung injury: a retrospective review of the ARDSnet tidal volume study cohort. J Intensive Care Med 2009;24(1):35-46. DOI: 10.1177/0885066608329850.

3. Hernández G, Teboul J-L. Fourth surviving sepsis campaign's hemodynamic recommendations: a step forward or a return to chaos? Crit Care 2017;21(1):133. DOI: 10.1186/s13054-017-1708-z.

4. Jozwiak M, Hamzaoui O, Monnet X, Teboul J-L. Fluid resuscitation during early sepsis: a need for individualization. Minerva Anestesiol 2018;84(8):987-992. DOI: 10.23736/S0375-9393.18.12422-9.

5. Osman D, Ridel C, Ray P, Monnet X, Anguel N, Richard C, et al. Cardiac filling pressures are not appropriate to predict hemodynamic response to volume challenge. Crit Care Med 2007;35(1):64-68. DOI: 10.1097/01.CCM.0000249851.94101.4F.

6. Mallat J, Meddour M, Durville E, Lemyze M, Pepy F, Temime J, et al. Decrease in pulse pressure and stroke volume variations after minifluid challenge accurately predicts fluid responsiveness. $\mathrm{Br} J$ Anaesth 2015;115(3):449-456. DOI: 10.1093/bja/aev222.

7. Boyd JH, Sirounis D, Maizel J, Slama M. Echocardiography as a guide for fluid management. Crit Care 2016;20(1):1-7. DOI: 10.1186/s13054016-1407-1.

8. Monnet X, Teboul JL. Assessment of volume responsiveness during mechanical ventilation: recent advances. Crit Care 2013:385-396. DOI: $10.1186 / \mathrm{cc} 12526$.

9. Weber U, Glassford NJ, Eastwood GM, Bellomo R, Hilton AK. A pilot assessment of carotid and brachial artery blood flow estimation using ultrasound doppler in cardiac surgery patients. J Cardiothorac Vasc Anesth 2016;30(1):141-148. DOI: 10.1053/j.jvca.2015.06.025.

10. Weber U, Glassford NJ, Eastwood GM, Bellomo R, Hilton AK. A pilot study of the relationship between Doppler-estimated carotid and brachial artery flow and cardiac index. Anaesthesia 2015;70(10): 1140-1147. DOI: 10.1111/anae.13069.

11. Chowhan G, Kundu R, Maitra S, Arora MK, Batra RK, Subramaniam R, et al. Efficacy of left ventricular outflow tract and carotid artery velocity time integral as predictors of fluid responsiveness in patients with sepsis and septic shock. Indian J Crit Care Med 2021;25(3): 310-316. DOI: 10.5005/jp-journals-10071-23764.

12. Sidor M, Premachandra L, Hanna B, Nair N, Misra A. Carotid flow as a surrogate for cardiac output measurement in hemodynamically stable participants. J Intensive Care Med 2020;35(7):650-655. DOI: 10.1177/0885066618775694.

13. Joshi M, Dhakane P, Bhosale SJ, Phulambrikar R, Kulkarni AP. Correlation between Carotid and Brachial Artery Velocity Time Integral and Their Comparison to Pulse Pressure Variation and Stroke Volume Variation for Assessing Fluid Responsiveness. Indian J Crit Care Med 2022;26(2):179-184. 